\title{
The Revelation Effect Occurs Whether or Not Working Memory Is Occupied
}

\author{
Hiroshi Miura*, Yuji Itoh \\ Keio University, Tokyo, Japan \\ Email: hiroshi9@f7.dion.ne.jp
}

How to cite this paper: Miura, H., \& Itoh, Y. (2018). The Revelation Effect Occurs Whether or Not Working Memory Is Occupied. Psychology, 9, 204-216.

https://doi.org/10.4236/psych.2018.92013

Received: December 20, 2017

Accepted: February 9, 2018

Published: February 12, 2018

Copyright $(9) 2018$ by authors and Scientific Research Publishing Inc. This work is licensed under the Creative Commons Attribution International License (CC BY 4.0).

http://creativecommons.org/licenses/by/4.0/

\begin{abstract}
The revelation effect is a phenomenon wherein performing a cognitive task before a recognition judgment induces "old" responses. One of the theories for the occurrence mechanism of the revelation effect is the criterion shift account (Niewiadomski \& Hockley, 2001). This account explains that because working memory is occupied when people solve a cognitive task, they adopt a more liberal criterion for recognition judgments immediately after a cognitive task than those with no preceding cognitive task. However, no studies of the revelation effect in which manipulation of working memory was intended have been conducted. We examined whether working memory load and capacity are related to the revelation effect. The results showed that neither the occurrence of the revelation effect nor its degree was affected by working memory load or capacity. As the results suggest working memory is not related to the revelation effect, a partially or entirely alternative account that can explain the revelation effect is needed.
\end{abstract}

\section{Keywords}

Recognition Judgment, Criterion Shift, Working Memory, Cognitive Process

\section{Introduction}

Performing a cognitive task before a recognition judgment induces "old" responses, this phenomenon is known as the revelation effect that was first demonstrated by Watkins and Peynircioglu (1990). They found that the old response rate of the recognition probes preceded by a revealed task (e.g., -1----n-, -1-p--n-, -l-p--nt, el-p--nt, el-p-ant, el-phant, elephant) was higher than those not preceded by the task.

Watkins and Peynircioğlu (1990) used the actual test stimulus in the preced*Faculty of Health Sciences, Kyorin University, Tokyo, Japan (Present Affiliation). 
ing cognitive task. For example, after conducting a revealed task using the word "elephant," participants made a recognition judgment of the word "elephant." However, Westerman and Greene (1996) showed the revelation effect using a test stimulus unrelated to that used in the preceding cognitive task. For example, after solving an anagram using the word "vineyard," participants made a recognition judgment of the word "elephant." It is thought that there are two types of revelation effect (Verde \& Rotello, 2004), and the former is called the "direct effect," whereas the latter is the "indirect effect" (Miura \& Itoh, 2016). In the present study we examined the indirect effect, because it was more suited to our experimental manipulation.

After Westerman and Greene (1996) showed the indirect effect, many researchers confirmed the effect using various cognitive tasks: letter-counting tasks, memory span tests, synonym-generation tasks (Westerman \& Greene, 1998), tasks of determining attractiveness ratings for inverted faces (Bornstein \& Wilson, 2004), articulatory suppression tasks (Miura \& Itoh, 2016), tasks of pressing arrow keys (Aßfalg, Currie, \& Bernstein, 2017), revealed tasks (Bornstein \& Neely, 2001; Westerman \& Greene, 1998), numerical addition tasks (Leynes, Landau, Walker, \& Addante, 2005; Niewiadomski \& Hockley, 2001), and anagram tasks (Aßfalg, Currie et al., 2017; Aßfalg \& Nadarevic, 2015; Azimian-Faridani \& Wilding, 2004; Bernstein, Rudd, Erdfelder, Godfrey, \& Loftus, 2009; Bernstein, Whittlesea, \& Loftus, 2002; Cameron \& Hockley, 2000; Hockley \& Niewiadomski, 2001; Kronlund \& Bernstein, 2006; Major \& Hockley, 2007; Miura \& Itoh, 2016; Niewiadomski \& Hockley, 2001; Verde \& Rotello, 2003, 2004; Westerman, 2000; Westerman, Miller, \& Lloyd, 2017; Young, Peynircioğlu, \& Hohman, 2009). While it has been shown that various cognitive tasks cause the revelation effect, few studies have found tasks that do not cause the effect. Digit span (Westerman \& Greene, 1998) and visual search tasks (Miura \& Itoh, 2016) did not cause the effect, but the reason for this is unclear. Whether quantity of the cognitive task affects the revelation effect or not is also unclear. Niewiadomski and Hockley (2001) examined whether a larger revelation effect was caused by double anagram tasks rather than by single anagram tasks. They found mixed results. The effect had the same size in the single and double task conditions in their Experiment 2, yet, the effect in the double task condition was larger than that in the single one in Experiment 4. In short, causation between quality and quantity of the cognitive tasks and the revelation effect is unclear. Since a lack of data prevents a detailed meta-analysis and revealing the mechanism of the effect (Aßfalg, Bernstein, \& Hockley, 2017), further research regarding the relationship between the cognitive task and the effect is needed.

Although occurrence mechanism of the revelation effect has not completely been clarified, there are several accounts explaining the mechanism. The criterion shift account (Niewiadomski \& Hockley, 2001) is one that is supported by various studies (for example, Miura \& Itoh, 2016; Verde \& Rotello, 2004). According to this account, when people solve a cognitive task, working memory is occupied. In a recognition test immediately after a cognitive task, because work- 
ing memory is occupied, they adopt a more liberal criterion for recognition judgments than in trials with no preceding cognitive task. Verde and Rotello (2004) indicated that the indirect effect of the revelation effect is induced by a response bias in recognition judgments, because conducting a cognitive task does not change the memory sensitivity of subsequent recognition. Miura and Itoh (2016) showed that an anagram or articulatory suppression task causes the revelation effect, but a visual search task does not. These results are consistent with the criterion shift account, because an anagram or an articulatory suppression task occupies working memory, whereas there is little occupation of working memory by a visual search task. Miura and Itoh (2016) remarked that the visual search task in the study was easy and placed little load on working memory. Therefore, the study is congruent with the criterion shift account; in that working memory occupation causes the revelation effect.

Although the criterion shift account includes concept of working memory in its explanation, no studies of the revelation effect involving intentional manipulation of working memory have been conducted. Working memory is a construct of memory that includes not only retention but also manipulation or control of information (Baddeley \& Hitch, 1974). There are many models of working memory: some have proposed subsystems of it (for example, Baddeley, 2000; Barnard, 1999), while others have emphasized its generality and unity (for example, Engle, Tuholski, Laughlin, \& Conway, 1999; Lovett, Reder, \& Lebiere, 1999). Although the extent to which generality and unity are emphasized differs between models, it is agreed that working memory has both a unitary and a non-unitary nature. The present study regards working memory as a general unity and does not consider its subsystems, because no studies of the revelation effect have intentionally manipulated working memory and we began by treating working memory as a general unity. If the present study shows a relation between the revelation effect and the general unity of working memory, then future research focusing on subsystems of working memory will be needed.

Westerman and Greene (1998) showed that both 3-letter and 8-letter memory span tests caused the revelation effect, and the degree of it did not differ between the two conditions. The result suggests that working memory load is not a critical factor for the revelation effect, because an 8-letter memory span test has a heavier load on working memory than a 3-letter one. However, it cannot be confidently concluded that working memory load is not related to the occurrence of the revelation effect, in that the number of letters in their experiment were three and eight. In their study, the correct answer rate in the 8-letter memory span tests was .07. Therefore, the working memory load in an 8-letter memory span test might be similar to that in a 3-letter one because the participants might not try to remember all the letters but partially remembered the first or last few letters in an 8-letter memory span test. Moreover, this might be the reason that 3and 8-letter memory span tests showed the revelation effect to the same degree, because a 3-letter memory span test had enough load on working memory to 
cause the effect. In sum, to demonstrate that working memory load does not affect the revelation effect, two other memory span tests that were not used in Westerman and Greene (1998) should be adopted as cognitive tasks. One test is a more than three- and less than eight-letter one, which is conducive to a relatively high correct answer rate. The other is a less than three-letter one, which does not have a heavier load than a 3-letter memory span test. Therefore, we used 5-letter memory span tests, considering that the limit for working memory capacity in humans are three to five chunks (Cowan, 2001). According to this, in a memory span test that does not allow participants to chunk the letters, 5 seems to be the maximum number of letters people can remember. To put a heavy cognitive load on participants without abandoning the effort to remember, 5-letter memory span tests were adopted. We also used 2-letter memory span tests. We should use less than 3-letter memory span tests, but a 1-letter memory span test does not occupy working memory but does occupy iconic memory. Thus, we used 2-letter ones.

In experimentally manipulating working memory load, individual differences in working memory capacity between participants should be considered. There are many studies in which working memory capacity was measured by a wellestablished task, for example, a counting span test (Case, Kurland, \& Goldberg, 1982), an operation span test (Turner \& Engle, 1989), and a reading span test (Daneman \& Carpenter, 1980). The reading span test was the oldest one measuring working memory capacity among these tests. In this test, while reading aloud a sentence, participants remember a target word in the sentence. Tests in languages other than English have also been developed. For example, in Japan, the Japanese reading span test (Osaka, 2002) is well-known as a test measuring working memory capacity. Although working memory capacity was measured in many studies, no research on the revelation effect measuring working memory capacity has been conducted. Taking individual working memory capacity into consideration enables more precise investigation of the effect of working memory load on the revelation effect.

The purpose of the present study was to examine whether working memory load and capacity is related to the revelation effect. We used 2- and 5-letter memory span tests as cognitive tasks to manipulate working memory load. A reading span test was also used in the present study. Measuring individual capacity for working memory may help to clarify the relation between the revelation effect and working memory. If working memory occupation affects the occurrence of the revelation effect, then the following results are predicted: 1) a larger revelation effect would be caused by 5-letter memory span tests than by 2-letter memory span tests (or the revelation effect would be caused only by 5-letter memory span tests). 2) A larger revelation effect would occur in participants with lower reading span than with higher reading span (or the revelation effect would occur only in participants with lower reading span). 3) A load-capacity interaction would be shown. For example, it would be predicted that both 2- and 5-letter 
memory span tests would cause the revelation effect in participants with lower reading span, but only 5-letter memory span tests would cause the revelation effect in participants with higher reading span. Alternatively, if working memory occupation does not affect the occurrence of the revelation effect, then none of the above-mentioned differences or any interactions would be shown.

\section{Method}

\subsection{Participants}

Forty-three undergraduate and graduate students participated in the experiment. They received 800 yen (approximately \$7), and were tested individually. Two participants were excluded from all analyses, because they had lived in Japan less than 10 years, and might be unsuitable for measuring working memory capacity using the Japanese reading span test. ${ }^{1}$ Another participant was also excluded from the analyses, because part of the data was not collected because of device trouble. The remaining 40 participants ( 14 males, 26 females) were aged between 19 and 24 years $(M=20.4)$. Participants provided informed consent, and the study was approved by the Research Ethics Committee at Keio University.

\subsection{Materials}

The study and test words were 108 four-letter nouns selected from a pool supplied by Amano and Kondo (1999). These words were written in Japanese katakana letters. The words were divided into two lists and buffers. Each list was composed of 48 words. One list was the "old list," which was presented both in the study phase and the test phase; the other was the "new list," which was presented only in the test phase. The old and new lists were counterbalanced across individuals. The buffers were presented as the first and last six trials during the study phase.

Twenty-one consonant and capital letters were used in the memory span tests. Two or five letters randomly selected from the 21 alphabetic items were used in a memory span test as a cognitive task. The selection was controlled so the same letter was not presented within a memory span test.

The Japanese reading span test (Osaka, 2002) was used as a task measuring working memory capacity in each participant. In the test, while reading aloud a sentence, participants remember a target word in the sentence. For example, after reading the sentences, "Since I missed the train, my mother drove me" and "He is gruff, but a nice guy at heart," recalling the underlined words was required (in the two-sentence phase). For the test in this experiment, five trials each were conducted from the two-sentence block to the five-sentence block. In sum, 20 trials were assigned, and 70 sentences were used in the test.

\subsection{Procedure}

The experiment was conducted in the following order: study phase, test phase,

${ }^{1} \mathrm{We}$ did not preset any criteria for participating in the experiment because almost all the students in Japan speak and read Japanese as the first language. However, two participants had lived for a shorter duration in Japan than in another country, and hence they were excluded from the analyses. 
and reading span test phase. In the study phase, 60 words were presented on a computer screen for $1 \mathrm{~s}$ each, with a $0.5 \mathrm{~s}$ blank interval between each word, after participants were instructed to remember these words. The order of words in the study phase was randomized for each participant.

At the beginning of the test phase, 5 practice trials of recognition judgments and memory span tests were conducted. In a recognition judgment, participants pressed the " 9 " key if they thought the word had been presented in the study phase (old responses) or pressed the " 0 " key if they thought the word had not been presented previously. After pressing the either key, they pressed the enter key to finalize the recognition judgment. In a memory span test, a letter was presented on the center of the computer screen for $950 \mathrm{~ms}$ each, with a $50 \mathrm{~ms}$ blank interval between each letter. After 2 or 5 letters were presented, the message, "Say!!" was presented on a screen. Then, participants recalled a series of letters orally in the presented order, and pressed the space key to finalize the memory span test. Additionally, they pressed the space key if they thought it was impossible to recall the letters. Two 2-letter memory span tests and three 5-letter memory span tests were conducted in the practice trials.

Then in the test phase, participants made recognition judgments with 48 old words and 48 new words. One-third of judgments (16 old and 16 new words) were preceded by a 2-letter memory span test (2-letter memory span test condition). Another third of judgments were preceded by a 5-letter memory span test (5-letter memory span test condition). The other third of judgments were not preceded by any memory span test (no memory span test condition). The order of presentation of stimuli and the matching between the words and the conditions in the test phase were randomized for each participant. Recollection of a series of letters and recognition judgments were self-paced, with a $1 \mathrm{~s}$ blank interval after pressing the enter or space key.

In the reading span test phase, while reading aloud a sentence, participants remember an underlined word in the sentence. They were instructed to read keeping a normal speed, with a distinct voice, and as accurately as possible. Immediately after participants finished reading a sentence, another sentence was presented, and they read it aloud again. This was repeated until the determined numbers of sentences were presented (for example, 5 sentences were presented in the five-sentence block). Then, a blank screen was presented, and participants orally recalled the underlined words in the correct order as much as possible. At the beginning of the reading span test phase, 2 practice trials of the 2 -sentence test were conducted. Then, the two-, three-, four-, and five-sentence blocks were conducted in this order. In each block, the determined-number-of-sentence tests were conducted for 5 trials. Participants had to recall the words in two-, three-, four-, and five-sentence tests within 10 s, 15 s, 20 s, and 25 s, respectively. An IC recorder was used to record responses to the reading span test and the memory span test.

\section{Results}

The mean correct response rate for the 2-letter memory span tests was 1.00 (SD 
$=0.06)$, and that for the 5-letter memory span tests was $0.78(S D=0.14)$. We considered only the responses whose letter-orders were exactly the same as the presented order to be correct. The mean correct response rate for the 5-letter memory span tests $(0.78)$ was significantly lower than that for the 2-letter memory span tests $(1.00), F(1,39)=89.28, M S e=0.010, p<0.001, \eta_{p}^{2}=0.70$. All recognition judgments were included in subsequent analyses (the judgments preceded by an incorrect response for the memory span test were not excluded), because it was thought that the revelation effect was not related to the feeling of resolution for the cognitive task preceded by a recognition judgment (Miura \& Itoh, 2016).

The mean proportions of old responses in word status and test conditions are summarized in Figure 1. A 2 (old, new) by 3 (5-letter memory span test, 2-letter memory span test, no memory span test) within-participants ANOVA was performed on the results. There was a significant main effect of word status, $F$ (1, $39)=254.30, M S e=0.042, p<0.001, \eta_{p}^{2}=0.87$. The proportion of old responses to old words was greater than that of old responses to new words. There was also a significant main effect of the test condition, $F(2,78)=5.18, M S e=$ $0.014, p<0.01, \eta_{p}^{2}=0.12$. The proportion of old responses in the 5 -letter memory span test condition and that in the 2-letter memory span test condition were greater than that in the no memory span test condition, $t(78)=3.02, p=$ $0.003, d=0.41, t(78)=2.47, p=0.02, d=0.34$, respectively. However, the proportion of old responses did not significantly differ between the 5- and 2-letter memory span test conditions, $t(78)=0.55, d=0.07$. An interaction between

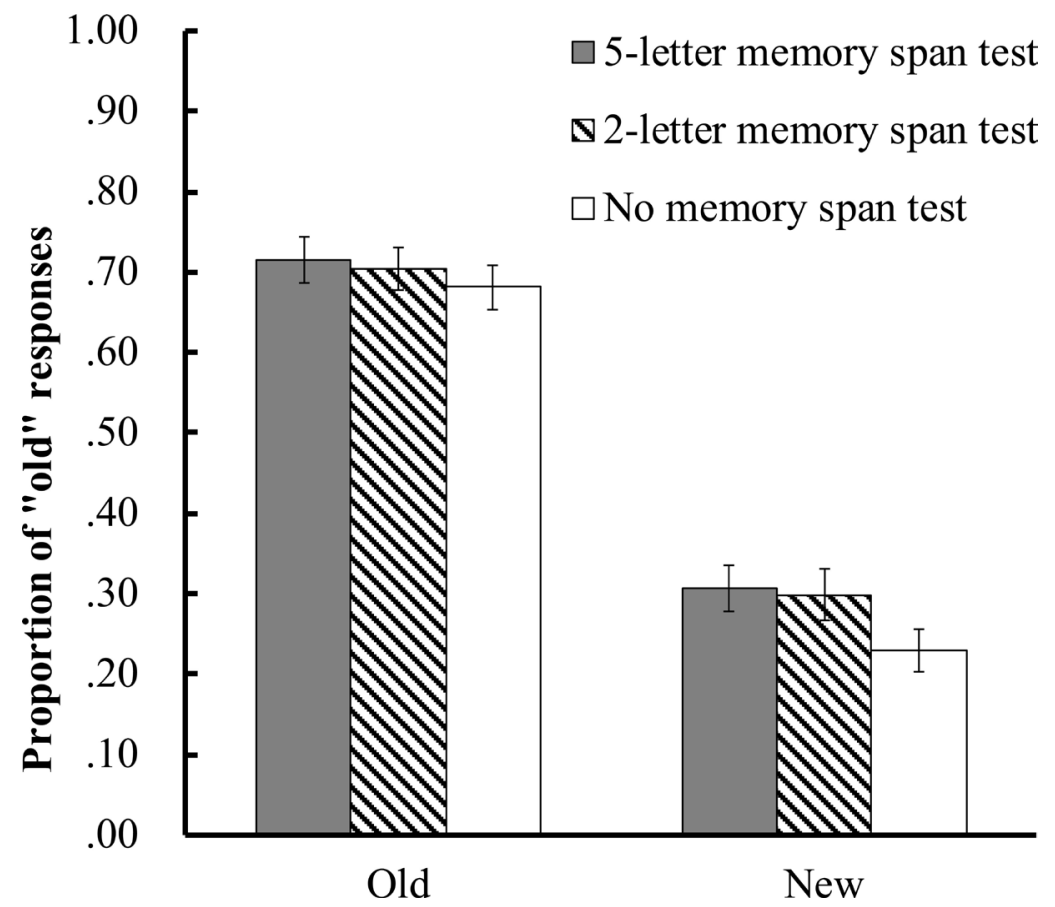

Figure 1. Proportions of "old" responses as a function of word status (old, new) and test condition (5-letter memory span test, 2-letter memory span test, no memory span test). Error bars represent standard errors. 
word status and test condition was not significant, $F(2,78)=1.08, M S e=0.013$, $\eta_{p}^{2}=0.03 .^{2}$

As the scoring procedure for the reading span test, we adopted the partialcredit unit scoring that had an advantage in that much information from the test was included (see, Conway et al., 2005). In this scoring, correct response rates for each trial was calculated (for example, if a participant recalled three words correctly in a four-sentence test, the correct response rate was 0.75), and those were averaged. Even if a participant recalled words in a different order, the words were regarded as correct.

The mean partial-credit unit score in the experiment was $0.64(S D=0.10)$. To examine whether the occurrence of the revelation effect was different between participants who had high scores and those who had low scores, 40 participants were divided into the two 20-participant groups: The high partial-credit unit score group (high reading span group) and the low partial-credit unit score group (low reading span group). The mean score in the former was 0.72 ( $S D=$ $0.06)$, and that in the latter was $0.56(S D=0.05)$. The mean proportions of old responses in word status, test conditions, and reading span are summarized in Table 1. A 2 (old, new) by 3 (5-letter memory span test, 2-letter memory span test, no memory span test) by 2 (high reading span, low reading span) mixed ANOVA was performed on the results. There was no significant main effect of reading span, $F(1,38)=0.15, M S e=0.096, \eta_{p}^{2}=0.00$. An interaction between reading span and test condition was not significant, $F(2,76)=0.63, M S e=$ $0.014, \eta_{p}^{2}=0.02$, and between reading span and word status was not significant, $F(1,38)=0.01, M S e=0.043, \eta_{p}^{2}=0.00$. Additionally, the three-way interaction was not significant, $F(2,76)=1.74, M S e=0.013, \eta_{p}^{2}=0.04$.

To examine the correlation between working memory capacity in each participant and the revelation effect, degrees of the revelation effect were calculated. The differences calculated by subtracting the old response rate in the no memory span test condition from that in the 2- or 5-letter memory span test condition were defined as the degrees of the revelation effect. There were no significant correlations between the partial-credit unit score in each participant and the degree of the revelation effect induced by the 2-letter memory span test $(r=0.07)$ and by the 5 -letter memory span test $(r=-0.08), t(38)=0.41, t(38)=-0.46$, respectively.

${ }^{2}$ We also conducted a 2 (old, new) by 3 (5-letter memory span test, 2-letter memory span test, no memory span test) ANOVA in which the judgments preceded by an incorrect response for the memory span test were excluded. The result was almost the same when they were not excluded. The proportion of old responses to old words was greater than that of old responses to new words, $F$ (1, $39)=246.93, M S e=0.044, p<0.001, \eta_{p}^{2}=0.86$. There was a significant main effect of the test condition, $F(2,78)=4.58, M S e=0.017, p<0.05, \eta_{p}^{2}=0.11$. The proportion of old responses in the 5-letter memory span test condition and that in the 2-letter memory span test condition were greater than that in the no memory span test condition, $t(78)=2.86, p=0.005, d=0.41, t(78)=2.28$, $p=0.03, d=0.34$, respectively. However, the proportion of old responses did not significantly differ between the 5 - and 2-letter memory span test conditions, $t(78)=0.58, d=0.08$. An interaction between word status and test condition was not significant, $F(2,78)=0.79, M S e=0.015, \eta_{p}^{2}=0.02$. 
Table 1. Proportions of "old" responses in all conditions, divided by performance on reading span test.

\begin{tabular}{ccccc}
\hline & \multicolumn{2}{c}{ High reading span group } & \multicolumn{2}{c}{ Low reading span group } \\
\hline Test condition & Old & New & Old & New \\
5-letter memory span test & $0.68(0.19)$ & $0.31(0.19)$ & $0.75(0.16)$ & $0.31(0.17)$ \\
2-letter memory span test & $0.71(0.18)$ & $0.28(0.19)$ & $0.70(0.15)$ & $0.32(0.21)$ \\
No memory span test & $0.69(0.17)$ & $0.22(0.18)$ & $0.67(0.18)$ & $0.24(0.16)$ \\
\hline
\end{tabular}

Note: Standard deviations are in parentheses.

\section{Discussion}

The purpose of the present study was to examine whether working memory load and capacity is related to the revelation effect. The results suggest that neither working memory load nor capacity affect the revelation effect. Considering each analysis in the study, we discuss the relation between working memory occupation and the revelation effect.

The correct response rate for the 2-letter memory span tests was 1.00 , and that for the 5-letter memory span tests was 0.78 . Considering the correct response rate for the 3-letter memory span test in Westerman and Greene (1998) almost reached perfection (0.95), the working memory load on the 2-letter memory span test in our experiment seemed to be light, because the load in the 2-letter one was lighter than that in the 3-letter one. Thus, when participants were engaged in the 2-letter memory span test, their working memory capacity seemed not to be fully used. Meanwhile, the working memory load in the 5-letter memory span test was relatively heavy, because the correct response rate for the 5-letter memory span tests was significantly lower than that for the 2-letter memory span tests. The fact that the rate for the 5-letter one was higher than that for the 8-letter one (0.07) in Westerman and Green (1998) also suggests the working memory load on the 5-letter one was relatively heavy. Regarding the reason for working memory load in the 5-letter test being relatively heavy, it is speculated that the moderately correct response rate in the 5-letter one indicates that participants always tried to remember all the letters, and sometimes could recall them all, while at other times they could not. These results suggest that the working memory load in our experiment was, to some extent, appropriately manipulated by changing the number of letters in the memory span tests.

If working memory load affects the revelation effect, 1) a larger revelation effect would be caused by 5-letter memory span tests than by 2-letter memory span tests. However, the results showed that the degree of the revelation effect did not differ between in the 2- and 5-letter memory span test conditions, although the effect did occur in both conditions. The results suggest that the revelation effect occurs without any relation to the degree of working memory load.

If working memory capacity is related to the occurrence of the revelation effect, 2) a larger revelation effect would occur in participants with lower reading span than with higher reading span. However, the results showed that the degree 
of the revelation effect in the high reading span condition did not differ from that in the low reading span condition. Moreover, the correlation between the degree of the revelation effect and the partial-credit unit score in each participant was not significant. We examined whether the degree of the revelation effect was negatively correlated with the partial-credit unit score, but did not find any correlations. Additionally, we did not find any interactions between the memory span test condition and the reading span condition; that is, 3) a loadcapacity interaction was not shown. These results consistently negated the relation between the revelation effect and working memory capacity. In sum, it is suggested that the revelation effect occurs regardless of working memory occupation.

The results of the present study were inconsistent with the criterion shift account (Niewiadomski \& Hockley, 2001), because this account assumes working memory occupation to be the cause of the revelation effect. There seems to be two possible explanations: One is that the criterion shift account is unsuitable for the revelation effect, the other is that working memory occupation does not cause the revelation effect, but a liberal criterion shift is induced by some other factor; that is, the account is partially suitable. In either case, an entirely or partially alternative account that can explain the occurrence mechanism of the revelation effect will be needed.

There are several limitations to the present study. In our experiment, only 2and 5-letter memory span tests were used as cognitive tasks. It is possible that working memory load determines whether the revelation effect occurs or not, but the boundary condition is outside the range of 2 to 5 letters. Considering the 8-letter memory span tests caused a revelation effect of the same size as the 3-letter ones and the correct response rate for the 8-letter ones was very low (0.07) in Westerman and Greene (1998), it is thought that examining more than 9-letter ones would hardly provide new findings. However, there is not a small possibility that the boundary condition of working memory load is less than 2 letters. Further research demonstrating that a cognitive task that rarely puts a load on working memory causes the revelation effect will be needed to clearly confirm that working memory occupation is not related to the revelation effect. Second, Type II errors might have occurred in our study. According to the null hypotheses, we considered that 2- and 5-letter memory span tests caused the revelation effect to the same degree, and this was what occurred in the high and the low reading span conditions. Replication will be needed to confirm whether our consideration is valid. Third, our experiment did not examine the validity of theories concerning the occurrence mechanism of the revelation effect except for the criterion shift account. Other than this account, the global matching theory (Westerman \& Greene, 1998), decrement to familiarity theory (Hicks \& Marsh, 1998), and discrepancy attribution theory (Whittlesea \& Williams, 2001) have been considered as theories for the revelation effect in a recent review of studies (Aßfalg, Bernstein et al., 2017). To examine the validity of these theories, Further 
research including variables in accordance with each theory will be needed.

Although there are several limitations, the present study seemed to make a modest contribution to studies of the revelation effect in that this is the first study in which working memory load and capacity were experimentally manipulated and these were not related to the occurrence of the revelation effect. To demonstrate new data for the revelation effect is very important, because a lack of data creates a serious obstacle to clarification of the mechanism of the revelation effect (Aßfalg, Bernstein et al., 2017). There is also a problem that few tasks that do not cause the revelation effect have been found (Miura \& Itoh, 2016). Considering the current state of studies of the revelation effect, many more studies of this effect should be performed, whether the effect occurs or not.

\section{Conclusion}

In the present study, the results suggest that working memory load and capacity were not related to the occurrence of the revelation effect. This is inconsistent with the criterion shift account that assumes the occupation of working memory causes the effect. An entirely or partially alternative account is needed because the mechanism of the revelation effect remains unclear. Further research that establishes the validity of the account is required.

\section{Acknowledgements}

We thank Kaori Endo for her advice for how to conduct the Japanese reading span test and how to analyze it.

\section{References}

Amano, S., \& Kondo, K. (1999). Lexical Properties of Japanese. Tokyo: Sanseido.

Aßfalg, A., \& Nadarevic, L. (2015). A Word of Warning: Instructions and Feedback Cannot Prevent the Revelation Effect. Consciousness and Cognition, 34, 75-86. https://doi.org/10.1016/j.concog.2015.03.016

Aßfalg, A., Bernstein, D. M., \& Hockley, W. (2017). The Revelation Effect: A Meta-Analytic Test of Hypotheses. Psychonomic Bulletin \& Review, 24, 1718-1741. https://doi.org/10.3758/s13423-017-1227-6

Aßfalg, A., Currie, D., \& Bernstein, D. M. (2017). Task Difficulty Moderates the Revelation Effect. Memory \& Cognition, 45, 664-676. https://doi.org/10.3758/s13421-016-0685-9

Azimian-Faridani, N., \& Wilding, E. L. (2004). An Event-Related Potential Study of the Revelation Effect. Psychonomic Bulletin \& Review, 11, 926-931. https://doi.org/10.3758/BF03196723

Baddeley, A. D. (2000). The Episodic Buffer: A New Component of Working Memory? Trends in Cognitive Sciences, 4, 417-423. https://doi.org/10.1016/S1364-6613(00)01538-2

Baddeley, A. D., \& Hitch, G. J. (1974). Working Memory. In G. H. Bower (Ed.), The Psychology of Learning and Motivation: Advances in Research and Theory (Vol. 8., pp. 47-89). New York, NY: Academic Press.

https://doi.org/10.1016/S0079-7421(08)60452-1 
Barnard, P. J. (1999). Interacting Cognitive Subsystems: Modeling Working Memory Phenomena within a Multiprocessor Architecture. In A. Miyake \& P. Shah (Eds.), Models of Working Memory: Mechanisms of Active Maintenance and Executive Control (pp. 298-339). New York, NY: Cambridge University Press. https://doi.org/10.1017/CBO9781139174909.012

Bernstein, D. M., Rudd, M. E., Erdfelder, E., Godfrey, R., \& Loftus, E. F. (2009). The Revelation Effect for Autobiographical Memory: A Mixture-Model Analysis. Psychonomic Bulletin \& Review, 16, 463-468. https://doi.org/10.3758/PBR.16.3.463

Bernstein, D. M., Whittlesea, B. W. A., \& Loftus, E. F. (2002). Increasing Confidence in Remote Autobiographical Memory and General Knowledge: Extensions of the Revelation Effect. Memory \& Cognition, 30, 432-438. https://doi.org/10.3758/BF03194943

Bornstein, B. H., \& Neely, C. B. (2001). The Revelation Effect in Frequency Judgment. Memory \& Cognition, 29, 209-213. https://doi.org/10.3758/BF03194914

Bornstein, B. H., \& Wilson, J. R. (2004). Extending the Revelation Effect to Faces: Haven't We Met before? Memory, 12, 140-146. https://doi.org/10.1080/09658210244000289

Cameron, T. E., \& Hockley, W. E. (2000). The Revelation Effect for Item and Associative Recognition: Familiarity versus Recollection. Memory \& Cognition, 28, 176-183. https://doi.org/10.3758/BF03213797

Case, R., Kurland, D. M., \& Goldberg, J. (1982). Operational Efficiency and the Growth of Short-Term Memory Span. Journal of Experimental Child Psychology, 33, 386-404. https://doi.org/10.1016/0022-0965(82)90054-6

Conway, A. R. A., Kane, M. J., Bunting, M. F., Hambrick, D. Z., Wilhelm, O., \& Engle, R. W. (2005). Working Memory Span Tasks: A Methodological Review and User's Guide. Psychonomic Bulletin \& Review, 12, 769-786. https://doi.org/10.3758/BF03196772

Cowan, N. (2001). The Magical Number 4 in Short-Term Memory: A Reconsideration of Mental Storage Capacity. Behavioral and Brain Sciences, 24, 87-114. https://doi.org/10.1017/S0140525X01003922

Daneman, M., \& Carpenter, P. A. (1980). Individual Differences in Working Memory and Reading. Journal of Verbal Learning and Verbal Behavior, 19, 450-466.

https://doi.org/10.1016/S0022-5371(80)90312-6

Engle, R. W., Tuholski, S. W., Laughlin, J. E., \& Conway, A. R. A. (1999). Working Memory, Short-Term Memory, and General Fluid Intelligence: A Latent-Variable Approach. Journal of Experimental Psychology: General, 128, 309-331. https://doi.org/10.1037/0096-3445.128.3.309

Hicks, J. L., \& Marsh, R. L. (1998). A Decrement-to-Familiarity Interpretation of the Revelation Effect from Forced-Choice Tests of Recognition Memory. Journal of Experimental Psychology: Learning, Memory, and Cognition, 24, 1105-1120. https://doi.org/10.1037/0278-7393.24.5.1105

Hockley, W. E., \& Niewiadomski, M. W. (2001). Interrupting Recognition Memory: Tests of a Criterion-Change Account of the Revelation Effect. Memory \& Cognition, 29, 1176-1184. https://doi.org/10.3758/BF03206387

Kronlund, A., \& Bernstein, D. M. (2006). Unscrambling Words Increases Brand Name Recognition and Preference. Applied Cognitive Psychology, 20, 681-687. https://doi.org/10.1002/acp.1220

Leynes, P. A., Landau, J., Walker, J., \& Addante, R. J. (2005). Event-Related Potential Evidence for Multiple Causes of the Revelation Effect. Consciousness and Cognition: An International Journal, 14, 327-350. https://doi.org/10.1016/j.concog.2004.08.005

Lovett, M. C., Reder, L. M., \& Lebiere, C. (1999). Modeling Working Memory in a Uni- 
fied Architecture: An ACT-R Perspective. In A. Miyake, \& P. Shah (Eds.), Models of Working Memory: Mechanisms of Active Maintenance and Executive Control (pp. 135-182). New York, NY: Cambridge University Press. https://doi.org/10.1017/CBO9781139174909.008

Major, J. C., \& Hockley, W. E. (2007). A Test of Two Different Revelation Effects using Forced-Choice Recognition. Psychonomic Bulletin \& Review, 14, 1096-1100. https://doi.org/10.3758/BF03193097

Miura, H., \& Itoh, Y. (2016). The Effect of the Feeling of Resolution and Recognition Performance on the Revelation Effect. Consciousness and Cognition, 45, 100-108. https://doi.org/10.1016/j.concog.2016.08.002

Niewiadomski, M. W., \& Hockley, W. E. (2001). Interrupting Recognition Memory: Tests of Familiarity-Based Accounts of the Revelation Effect. Memory \& Cognition, 29, 1130-1138. https://doi.org/10.3758/BF03206382

Osaka, M. (2002). Working Memory: The Sketchpad in the Brain. Tokyo: Shinyosha.

Turner, M. L., \& Engle, R. W. (1989). Is Working Memory Capacity Task Dependent? Journal of Memory and Language, 28, 127-154. https://doi.org/10.1016/0749-596X(89)90040-5

Verde, M. F., \& Rotello, C. M. (2003). Does Familiarity Change in the Revelation Effect? Journal of Experimental Psychology: Learning, Memory, and Cognition, 29, 739-746. https://doi.org/10.1037/0278-7393.29.5.739

Verde, M. F., \& Rotello, C. M. (2004). ROC Curves Show That the Revelation Effect Is Not a Single Phenomenon. Psychonomic Bulletin \& Review, 11, 560-566. https://doi.org/10.3758/BF03196611

Watkins, M. J., \& Peynircioğlu, Z. F. (1990). The Revelation Effect: When Disguising Test Items Induces Recognition. Journal of Experimental Psychology: Learning, Memory, and Cognition, 16, 1012-1020. https://doi.org/10.1037/0278-7393.16.6.1012

Westerman, D. L. (2000). Recollection-Based Recognition Eliminates the Revelation Effect in Memory. Memory \& Cognition, 28, 167-175. https://doi.org/10.3758/BF03213796

Westerman, D. L., \& Greene, R. L. (1996). On the Generality of the Revelation Effect. Journal of Experimental Psychology: Learning, Memory, and Cognition, 22, 1147-1153. https://doi.org/10.1037/0278-7393.22.5.1147

Westerman, D. L., \& Greene, R. L. (1998). The Revelation That the Revelation Effect Is Not Due to Revelation. Journal of Experimental Psychology: Learning, Memory, and Cognition, 24, 377-386. https://doi.org/10.1037/0278-7393.24.2.377

Westerman, D. L., Miller, J. K., \& Lloyd, M. E. (2017). Revelation Effects in Remembering, Forecasting, and Perspective Taking. Memory \& Cognition, 45, 1002-1013. https://doi.org/10.3758/s13421-017-0710-7

Whittlesea, B. W. A., \& Williams, L. D. (2001). The Discrepancy-Attribution Hypothesis: I. The Heuristic Basis of Feelings and Familiarity. Journal of Experimental Psychology: Learning, Memory, and Cognition, 27, 3-13. https://doi.org/10.1037/0278-7393.27.1.3

Young, K. D., Peynircioğlu, Z. F., \& Hohman, T. J. (2009). Revelation Effect in Metamemory. Psychonomic Bulletin \& Review, 16, 952-956.

https://doi.org/10.3758/PBR.16.5.952 